\title{
EDITORIAL
}

\section{Crystalline porous materials: from zeolites to metal-organic frameworks (MOFs)}

\author{
Zaiku Xie $(\bowtie)^{1}$, Bao-Lian Su $(\bowtie)^{2,3}$ \\ 1 State Key Laboratory of Green Chemical Engineering and Industrial Catalysis, Sinopec Shanghai Research Institute of Petrochemical Technology, \\ Shanghai 201208, China \\ 2 Laboratory of Inorganic Materials Chemistry (CMI), University of Namur, Namur, Belgium \\ 3 State Key Laboratory of Advanced Technology for Materials Synthesis and Processing, Wuhan University of Technology, Wuhan 430074, China
}

(C) Higher Education Press and Springer-Verlag GmbH Germany, part of Springer Nature 2020

As a class of important crystalline porous materials, zeolites which were first found in 1756 have now been widely used in chemical industries for catalysis, adsorption and separation. 35232 patents with the title including "zeolite*" are documented by Derwent Innovations Index on January 2, 2020 [1]. Despite of spread applications of zeolites and related-materials in industry, fundamental research of zeolites and their applications are still desired in both academia and industry, as shown in Fig. 1. Roughly 30271 publications with "zeolite*" in titles are recorded by the Web of Science Core Collection on January 2, 2020 [2]. Among the top 10 organizations with the large number of publications on "zeolite", six (6) are from China, namely Chinese Academy of Sciences (Chinese Acad Sci), Dalian University of Technology (Dalian Univ Technol), Jilin University (Jilin Univ), Zhejiang University (Zhejiang Univ), China University of Petroleum (China Univ Petr) and Fudan University (Fudan Univ), as shown in Table 1.

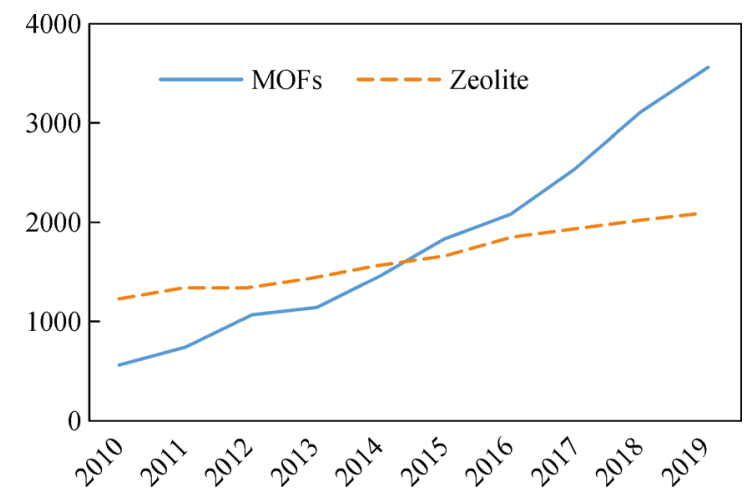

Fig. 1 Publications with Zeolite/MOFs in titles collected by the Web of Science Core Collection on January 2, 2020.

Regarding the application of zeolites in heterogeneous catalysis, it is very important to develop controllable green synthesis methods, to unveil the relationship between structure and catalytic performance, to design and prepare new zeolites with versatile topologies, and to explore new catalytic reactions. In this Special Issue we collected three (3) Reviews and four (4) Research Articles. Inayat et al. at the University of Erlangen-Nuremberg presented a comparative review on the state-of-the-art of various preparation routes of layer-like FAU-type zeolites [3]. Tang et al. reviewed the recent progress of the seed-induced synthesis of functional MFI zeolites [4]. Deng et al. employed the

Received January 15, 2020

E-mails: xzk@sinopec.com (Xie Z), bao-lian.su@unamur.be (Su BL) 
Table 1 Top 10 organizations with the peer-reviewed publications on the "zeolite" and "MOFs" according to the Web of Science Core Collection on January 2, 2020

\begin{tabular}{|c|c|c|c|c|c|c|}
\hline \multirow{2}{*}{ No. } & \multicolumn{3}{|c|}{ Zeolite } & \multicolumn{3}{|c|}{ MOFs } \\
\hline & Organizations & Publications & Percentage & Organizations & Publications & Percentage \\
\hline$\overline{1}$ & Chinese Acad Sci & 1052 & $3.47 \%$ & Chinese Acad Sci & 1276 & $6.47 \%$ \\
\hline 2 & Russian Acad Sci & 615 & $2.03 \%$ & Northwestern Univ & 567 & $2.87 \%$ \\
\hline 3 & Univ Politecn Valencia & 487 & $1.60 \%$ & Univ Calif Berkeley & 427 & $2.17 \%$ \\
\hline 4 & Dalian Univ Technol & 356 & $1.18 \%$ & Nankai Univ & 420 & $2.14 \%$ \\
\hline 5 & Jilin Univ & 355 & $1.17 \%$ & Jilin Univ & 397 & $2.02 \%$ \\
\hline 6 & Katholieke Univ Leuven & 331 & $1.09 \%$ & Nanjing Univ & 383 & $1.95 \%$ \\
\hline 7 & Zhejiang Univ & 293 & $0.97 \%$ & Univ Chinese Acad Sci & 330 & $1.68 \%$ \\
\hline 8 & China Univ Petr & 286 & $0.95 \%$ & Zhejiang Univ & 318 & $1.61 \%$ \\
\hline 9 & Fudan Univ & 267 & $0.88 \%$ & Texas A M Univ & 309 & $1.57 \%$ \\
\hline 10 & Univ Tokyo & 265 & $0.88 \%$ & King Abdulaziz Univ & 236 & $1.19 \%$ \\
\hline
\end{tabular}

solid-state NMR to establish the correlation between structure and property of active sites in metal-containing zeolites, providing a better mechanistic understanding of the catalytic properties of related materials [5]. The novel synthesis methods for mesoporous SSZ-39 [6], mesoporous Y zeolites [7], hierarchical ZSM-5 zeolites with radial mesopores [8] and core/shell structures [9] are also reported in this Special Issue. All the prepared zeolites exhibit better catalytic performance. For example, the mesoporous SSZ-39 exhibits the enhanced catalyst lifetime in methanol-to-olefins (MTO) reaction [6]; the mesoporous $Y$ zeolites obtained by the ultrasound irradiation method showed the improved acidic property, as well as the good catalytic activity in catalytic cracking of $n$-octane [7]; hierarchical ZSM-5 zeolites with radial mesopores demonstrated good catalytic activity and longevity in alkylation of benzene [8]; and hierarchical core/shell ZSM-5 showed excellent activity in the ketalation and acetalization reactions [9].

Analogous to zeolites, metal-organic frameworks (MOFs) are another class of crystalline porous materials with highly versatile and flexible structures and functionalities, which are now star materials for many applications including separation and catalysis. As a relatively new class of porous materials, fundamental research on MOFs is flourishing (refer to numbers of publications related to MOFs, Fig. 1). In 2017, Prof. Zhou of Peking University and Prof. Zhao of Nanyang Technological University highlighted recent research advancements in zeolites, ordered mesoporous silica and MOFs for heterogeneous catalysis, which inspired further research into this rapidly developing field [10]. In addition to catalysis, other major potential applications of MOFs include gas storage and separation, sensing, drug delivery, etc., as shown in Fig. 2. Xinlei Liu reviewed five approaches for fabricating UiO-66 polycrystalline membranes and their applications in gas separation, pervaporation, nanofiltration and ion separations [11]. MOFs-based processes for $\mathrm{CO}_{2}$ capture may address the current issues related to the greenhouse gas emissions. Yang and Ban developed a conceptional framework from material design and membrane separation application for $\mathrm{CO}_{2}$ capture, emphasizing two important yet promising themes [12]. Relevant works on MOFs from Iranian colleagues were also collected by this Special Issue [13].

Fundamental and applied research related to zeolites and MOFs will see continuous growth in the future due to their unique properties and practical applications in the real world, as well as the unrealized or unexplored potentials for other emerging applications. In this Special Issue, we only collected a few works contributed by relevant colleagues, which only reflect a small proportion of the past and current research of the two materials. However, we still wish that this Special Issue will provide a timely summary of the research fields, contributing to the further progress of the research into the two classes of porous crystalline materials. At the end, we are grateful for all the contributing authors, reviewers and editors for this Special Issue, their time, effort and dedication are much appreciated. And we also appreciate the help for the organization of Yangdong Wang, Chuanming Wang, Yujue Du, and Lihua Chen. 


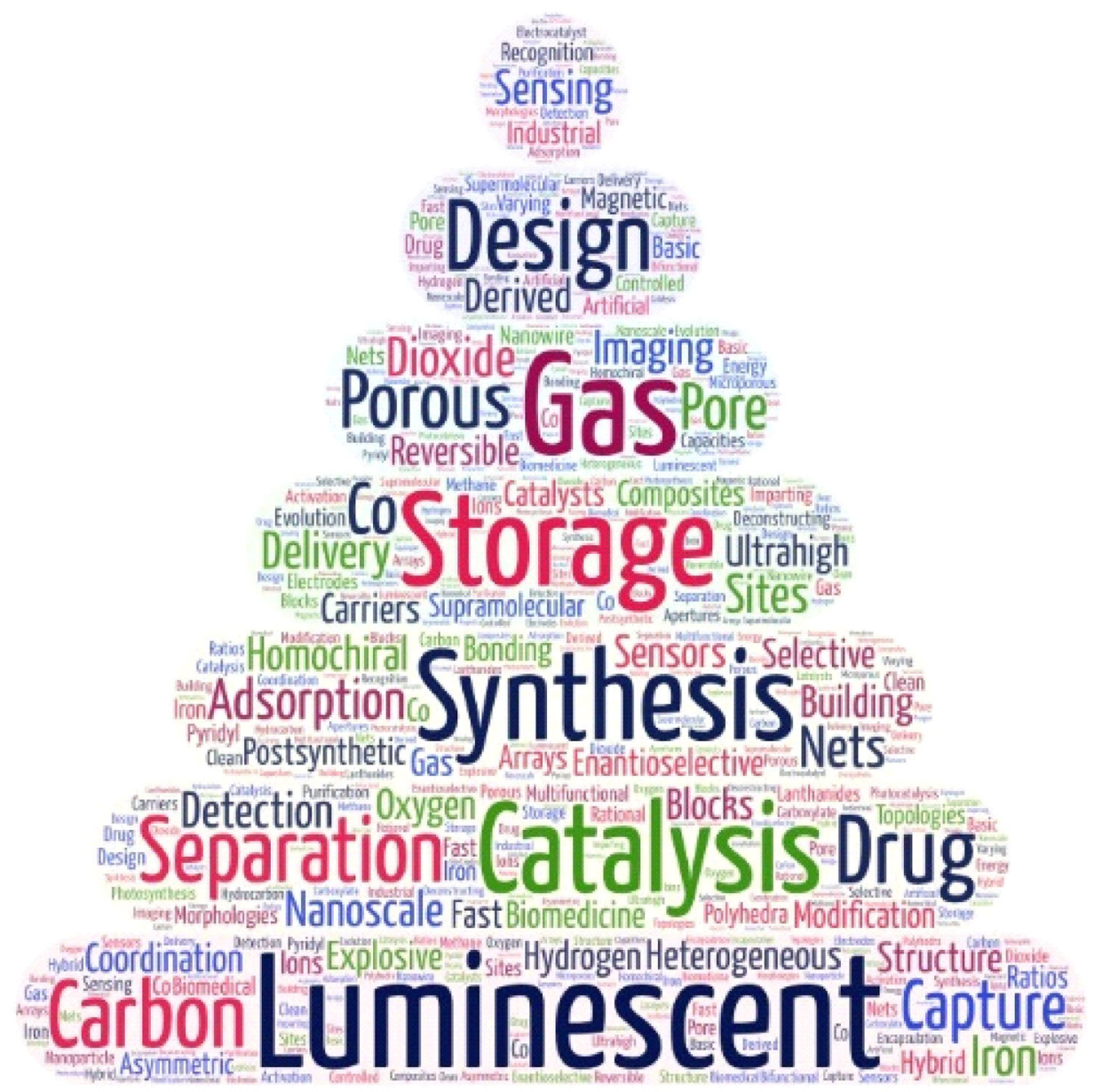

Fig. 2 Word cloud based on the top 50 high-cited papers with "Metal-Organic Frameworks or MOFs" in titles from the database Web of Science Core Collection on January 2, 2020.

\section{References}

1. Derwent Innovations Index. Web of Science website, 2020

2. Web of Science core collection. Web of Science website, 2020

3. Reiprich B, Weissenberger T, Schwieger W, Inayat A. Layer-like FAU-type zeolites: A comparative view on 7 different preparation routes. Frontiers of Chemical Science and Engineering, 2020, 14(2): 127-142

4. Ye Z Q, Zhang H B, Zhang Y H, Tang Y. Seed-induced synthesis of functional MFI zeolite materials: Method development, crystallization mechanism, and catalytic property. Frontiers of Chemical Science and Engineering, 2020, 14(2): 143-158

5. Zhao X L, Xu J, Deng F. Solid-state NMR for metal-containing zeolites: From active site to reaction mechanism. Frontiers of Chemical Science and Engineering, 2020, 14(2): 159-187

6. Xu H, Lei C, Wu Q M, Zhu Q Y, Meng X J, Dai D, Maurer S, Parvulescu A, Müller U, Xiao F S. Organosilane surfactant-assisted synthesis of mesoporous SSZ-39 zeolite with good catalytic performance in methanol-to-olefins (MTO) reaction. Frontiers of Chemical Science and Engineering, 2020, 14(2): 267-274

7. Zhang R X, Zhong P N, Arandiyan H, Guan Y N, Liu J M, Wang N, Jiao Y T, Fan X L. Using ultrasound to improve the sequential post-synthesis modification method for making mesoporous Y zeolites. Frontiers of Chemical Science and Engineering, 2020, 14(2): 275-287

8. Wang D R, Sun H M, Liu W, Shen Z H, Yang W M. Hierarchical ZSM-5 zeolite with radial mesopores: Preparation, formation mechanism and application in benzene alkylation. Frontiers of Chemical Science and Engineering, 2020, 14(2): 248-257

9. Luo P, Guan Y J, Xu H, He M Y, Wu P. Postsynthesis of hierarchical core/shell ZSM-5 as efficient catalyst in ketalation and acetalization reactions. Frontiers of Chemical Science and Engineering, 2020, 14(2): 258-266

10. Liang J, Liang Z B, Zou R Q, Zhao Y L. Heterogeneous catalysis in zeolites, mesoporous silica, and metal-organic frameworks. Advanced 
Materials, 2017, 29(30): 1701139

11. Liu X L. Metal-organic framework UiO-66 membranes. Frontiers of Chemical Science and Engineering, 2020, 14(2): 216-232

12. Ban Y J, Zhao M, Yang W S. Metal-orgnic framework-based $\mathrm{CO}_{2}$ capture: From precise material design to high-efficiency membranes. Frontiers of Chemical Science and Engineering, 2020, 14(2): 188-215

13. Pirzadeh K, Ghoreyshi A A, Rahimnejad M, Mohammadi M. Optimization of electrochemically synthesized $\mathrm{Cu}_{3}(\mathrm{BTC})_{2}$ by Taguchi method for $\mathrm{CO}_{2} / \mathrm{N}_{2}$ separation and data validation through artificial neural network (ANN) modeling. Frontiers of Chemical Science and Engineering, 2020, 14(2): 233-247

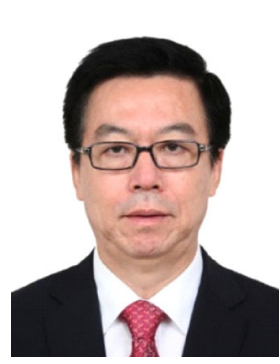

Professor Zaiku Xie received his PhD degree from East China University of Science and Technology. He was elected academician of the Chinese Academy of Sciences in 2017. He is now the director of the science and technology department of SINOPEC and the executive deputy director of the science and technology committee of SINOPEC. He is now the vice president of the Catalysis Society of China from 2012, the vice director of Chinese Chemical Society from 2019, and the member of the Committee on Chemistry and Industry (COCI) of IUPAC from 2014. He was elected as a fellow of the Royal Society of Chemistry in 2013. His research interests focus on the preparation, characterization, and industrially catalytic conversion of novel zeolite catalysts. He has won many important awards, such as the first prize of National Science \& Technology Progress Award, second prize of National Technology Invention Award, Gold Medal of Chinese Patent, and Ho Leung Ho Lee Prize for Scientific and Technological Innovation.

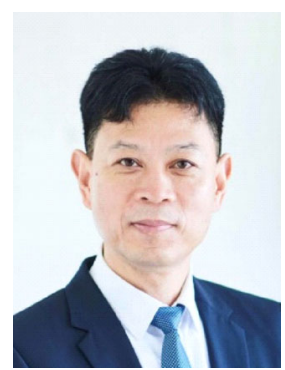

Bao-Lian Su is a member of the Royal Academy of Belgium and the European Academy of Sciences, a fellow of the Royal Society of Chemistry and a life member of Clare Hall College, University of Cambridge. He holds "Chaire Francqui au titre Belge". He joined the faculty at the University of Namur and created the Laboratory of Inorganic Materials Chemistry (CMI) in 1995. He is currently a Director of the Laboratory of Inorganic Materials Chemistry (CMI), University of Namur, Belgium. He is a "Changjiang Professor" and a "Strategical Scientist" at the Wuhan University of Technology. He has received a series of honours and awards such as the First Prize Invention Award of Sinopec (1992, China), A. Wetrems Prize (2007, Royal Academy of Belgium) and IUPAC Distinguished Award for Novel Materials and their synthesis (2011). His current research fields include the synthesis, the property study and the molecular engineering of organized, hierarchically porous and bio-inspired materials, biomaterials, living materials, leaf-like materials and nanostructures and the immobilisation of living organisms for artificial photosynthesis, nanotechnology, biotechnology, cell therapy and biomedical applications. 